\title{
SÍNDROME DE MOEBIUS EM PACIENTE COM FISSURA LABIOPALATINA: RELATO DE CASO
}

\section{MOEBIUS SYNDROME IN PATIENTS WITH CLEFT LIP AND PALATE: CASE REPORT}

\author{
André Victor Pinto Serra', Carlos Vinícius Ayres Moreira' ${ }^{2}$, Roberto Almeida de Azevedo ${ }^{3}$ \\ Nilmara Dias Santos ${ }^{4}$, Larissa Oliveira Ramos Silva ${ }^{5}$
}

'Graduado em Odontologia. Residente do Serviço de Cirurgia e Traumatologia Bucomaxilofacial da Universidade Federal da Bahia/ Obras Sociais Irmã Dulce. Salvador, Bahia, Brasil. andreserra3@gmail.com ${ }^{2}$ Graduado em Odontologia. Residente do Serviço de Cirurgia e Traumatologia Bucomaxilofacial da Universidade Federal da Bahia/ Obras Sociais Irmã Dulce. Salvador, Bahia, Brasil.cv_amoreira@yahoo.com.br ${ }^{3}$ Doutor em Odontologia. Docente na Universidade Federal da Bahia. Coordenador do Serviço de Cirurgia e Traumatologia Bucomaxilofacial da Universidade Federal da Bahia/Obras Sociais Irmã Dulce. Salvador, Bahia, Brasil. razevedo@ufba.br Autora para correspondência. Cirurgiã-dentista no Centro de Especialidades Odontológicas I. Alagoinhas, Bahia, Brasil.nildias1 $1 @$ gmail.com ${ }^{5}$ Graduada em Odontologia pela Universidade Federal da Bahia. Ex-interna do Serviço de Cirurgia e Traumatologia Bucomaxilofacial da Universidade Federal da Bahia/Obras Sociais Irmã Dulce. larissaramost@hotmail.com

RESUMO | A síndrome de Moebius ou sequência de Moebius (SM), desordem congênita rara, não progressiva, de severidade variada, tem como principal manifestação paralisia uni ou bilateral do nervo facial, que confere inatividade dos músculos da expressão facial por ele inervados. As manifestações orofaciais que acompanham a SM são fissura palatina, língua fissurada, tônus dos músculos faciais e da língua deficiente, hipoplasia dentária, sialorreia por ausência de selamento labial, cárie, doença periodontal, micrognatia, microstomia, mordida aberta, úvula bífida, dificuldade da realização de movimentos excursivos mandibulares, pequenas fissuras palpebrais, ptose palpebral, epicanto bilateral, hipertelorismo ocular, deformidade da orelha externa com perda ocasional da audição. Portadores de SM com paralisia facial bilateral apresentam incapacidade para sorrir, devido à ausência de movimentos de elevação dos lábios e da comissura labial, além de dificuldades na fala, mastigação e deglutição. $O$ objetivo deste estudo é relatar a abordagem terapêutica em paciente de 08 anos de idade portador de fissura pós-forame associada à Síndrome de Moebius atendido no Serviço de Cirurgia Bucomaxilofacial do centro de fissurados das Obras Sociais Irmã Dulce (Salvador, Bahia), que apresentou ausência de expressão facial, déficit motor ocular, ausência de selamento labial com sialorreia, microstomia, hiperplasia gengival generalizada e dentes decíduos cariados. A paralisia facial e as malformações orofaciais e musculoesqueléticas apresentadas por portadores da síndrome de Moebius têm uma série de implicações sobre a saúde geral e bucal. A abordagem precoce do cirurgião-dentista é fundamental para o estabelecimento de uma condição bucal que garanta melhor qualidade de vida ao paciente.

\begin{abstract}
The Moebius syndrome or sequence of Moebius (MS), a rare, non-progressive congenital disorder of varying severity, its main manifestation unilateral or bilateral paralysis of the facial nerve, which gives inactivity of the muscles of facial expression for him innervated. Orofacial manifestations of MS are cleft palate, fissured tongue and tone the facial muscles and poor language, dental hypoplasia, drooling by the absence of lip sealing, caries, periodontal disease, micrognathia, microstomia, open bite, bifid uvula, difficulty performing excursive mandibular movements, small palpebral fissures, ptosis, bilateral epicanthus, ocular hypertelorism, deformity of the outer ear with occasional hearing loss. Patients with MS with bilateral facial paralysis present inability to smile, due to the absence of lifting the lips and labial movements, and difficulties with speech, chewing and swallowing. The aim of this study is to report the therapy in 08 years of carrier old cleft patient post foramen approach associated with Moebius syndrome attended the Oral and Maxillofacial Surgery Department of fissured center Obras Sociais Irmã Dulce (Salvador, Bahia), which showed no facial expression, eye motor deficit, absence of lip seal with drooling, microstomia, generalized gingival hyperplasia and decayed deciduous teeth. Facial paralysis and orofacial and musculoskeletal malformations presented by patients with Moebius syndrome has a number of implications for the general and oral health. The early approach of the dentist is important to the establishment of an oral condition that ensures better quality of life to the patient.
\end{abstract}

Keywords: möebius syndrome, cleft palate, facial nerve.

Palavras-chave: síndrome de möebius; fissura palatina; nervo facial. 


\section{INTRODUÇÃO}

A Síndrome de Moebius ou Sequência Moebius (SM), é uma desordem congênita rara, não progressiva, de severidade variada, caracterizada pela paralisia unilateral ou bilateral dos nervos cranianos facial (VII) e abducente (VI). A área por eles inervada tem atividade muscular comprometida, sendo observados ausência de movimentos de lateralidade ocular e de expressões da mímica facial. ${ }^{1-11}$ A prevalência é estimada em 1:250.000 nascidos vivos, com igual incidência sobre os sexos e a maioria dos casos são de acometimento esporádico, sendo apenas $2 \%$ relacionados ao histórico familiar. ${ }^{7}$

Descrita pela primeira vez por Albretch Von Graefe em 1880 e associada com outras malformações por Paul Julius Moebius em 1888, a SM é diagnosticada pelos seguintes critérios: paralisia completa ou parcial do nervo facial; malformações dos membros (sindactilia, braquidactilia ou agenesia de dedos, e pés tortos) presentes com frequência; associação da paralisia do nervo facial completa ou parcial com paralisia do nervos abducente (VI), oculomotor (III) e troclear (IV); hipoplasia da língua devido à paralisia do nervo hipoglosso (XII); dificuldade na fonação e deglutição pela paralisia dos nervos cranianos trigêmeo (V), glossofaríngeo (IX) e vago $(\mathrm{X})$; deformidades orofaciais e musculoesqueléticas. ${ }^{5}$ O diagnóstico é alcançado ainda nos primeiros anos de vida, quando já é possível observar o incompleto fechamento da pálpebra durante o sono, a dificuldade de sucção e a ausência do sorriso e de expressões faciais, mesmo com o choro. ${ }^{5,7,9}$

Com etiologia não completamente esclarecida, a hipótese mais aceita é que a SM pode ser causada por hipóxia/isquemia das células neurais do feto, decorrente da interrupção do suprimento vascular durante $\circ$ primeiro trimestre da gestação. Este fenômeno pode ser provocado por uma contração uterina estimulada por fármacos ou agentes tóxicos, como o misoprostol, benzodiazepínicos, talidomida, álcool e cocaína. Outros possíveis fatores etiológicos são: rubéola durante a gravidez, diabetes gestacional, hipertermia, distúrbio no desenvolvimento vascular romboencefálico determinado por alterações genéticas e relações consanguíneas. ${ }^{1}, 2,3,5,7,8,9$
Os pacientes com a SM podem apresentar malformações musculoesqueléticas como ausência da cabeça esternal do músculo peitoral maior associação com síndrome de Poland, defeitos em costelas, artrogripose peitoral e até dextrocardia, pés tortos, sindactilia, braquidactilia ou agenesia de dedos das mãos e dos pés e distúrbios neurológicos, como epilepsia e retardo cognitivo, que ocorrem em cerca de $15 \%$ dos casos. ${ }^{5,11}$ As manifestações orofaciais que acompanham a SM são: fissura palatina, língua fissurada, tônus dos músculos faciais e da língua deficiente, hipoplasia dentária, sialorreia por ausência de selamento labial, cárie, doença periodontal, micrognatia, microstomia, mordida aberta, úvula bífida, dificuldade da realização de movimentos excursivos mandibulares, pequenas fissuras palpebrais, ptose palpebral, epicanto bilateral, hipertelorismo ocular, deformidade da orelha externa com perda ocasional da audição. ${ }^{1,2,3,8}$ Portadores de SM com paralisia facial bilateral apresentam incapacidade para sorrir, devido à ausência de movimentos de elevação dos lábios e da comissura labial, além de dificuldades na fala, mastigação e deglutição. Quando a paralisia é unilateral, a mímica facial é distorcida e compensada para o lado não afetado. ${ }^{1,2,5,10}$

As implicações da SM sobre a capacidade visual, motora, cognitiva, nutricional, comunicativa e social dos seus portadores exige um tratamento multidisciplinar e com acompanhamento periódico. Muitos sinais e sintomas da SM estão relacionados à região bucomaxilofacial, portanto a atuação do cirurgião-dentista é indispensável para a prevenção de patologias decorrentes da dificuldade de higienização bucal, como a cárie e a gengivite, a correção de maloclusões e o restabelecimento da mímica facial.

objetivo deste estudo é relatar a abordagem terapêutica em paciente portador de SM atendido no Serviço de Cirurgia Bucomaxilofacial do centro de fissurados das Obras Sociais Irmã Dulce (Salvador, Bahia). 


\section{RELATO DE CASO}

Paciente do sexo masculino, 08 anos de idade, portador de fissura pós-forame associada à Síndrome de Moebius, e outros diagnósticos como atraso de desenvolvimento psicomotor e cognitivo, em atendimento multidisciplinar no Centrinho, setor de referência em atendimento a pacientes fissurados do Hospital Santo Antônio (Obras Sociais Irmã Dulce, Salvador, Bahia) foi encaminhado à equipe de Cirurgia Bucomaxilofacial devido à necessidade de exodontias. Responsável pela criança afirma não ter histórico familiar da SM, nem parentes com manifestações semelhantes. Genitora relata gestação com intercorrências, dentres elas sangramento uterino e oligoidrâmnio (perda de líquido anminiótico), nega consanguinidade, uso de bebidas alcoólicas e tabagismo durante gestação e lactação. O parto foi do tipo cesárea com 32 semanas de gestação, a criança nasceu com 965 gramas, evoluiu com icterícia tardia e conjuntivite, necessitando de internamento por 02 meses. Foi observado hipertelorismo ocular, retrognatia, micrognatia, pé torto à direita. Em testes de audição foi acusada perda parcial de audição bilateral.
Apresenta características típicas da síndrome de Moebius, como ausência de expressão facial, déficit motor ocular, ausência de selamento labial com sialorréia (Figuras 1 e 2). Ao exame intraoral, notase hiperplasia gengival generalizada, presença de cárie nas unidades decíduas 6.2, 6.4, 7.2, 7.4 e 7.5, fissura pós-forame abordada em outra unidade hospitalar. Além de não ser colaborativo a procedimentos dentários, paciente apresenta abertura bucal limitada devido à microstomia. Ao exame de imagem observa-se dentição mista, ausência de dentes supranumerários ou impactados (Figura 3). Optou-se por instituir o tratamento sob anestesia geral em ambiente hospitalar, sendo realizada exodontia de cinco unidades decíduas comprometidas por cárie e raspagem supragengival (Figuras 4 e 5). Em acompanhamento, evolui com melhora da condição do meio bucal. Paciente e familiares receberam orientações sobre higiene oral e prevenção de lesões de cárie futuras, através de consultas periódicas com o odontopediatra. $O$ presente caso foi aprovado pelo Comitê de Ética em Pesquisa da Faculdade de Odontologia da Universidade Federal da Bahia sob parecer CAAE: 68065417.1.0000.5024

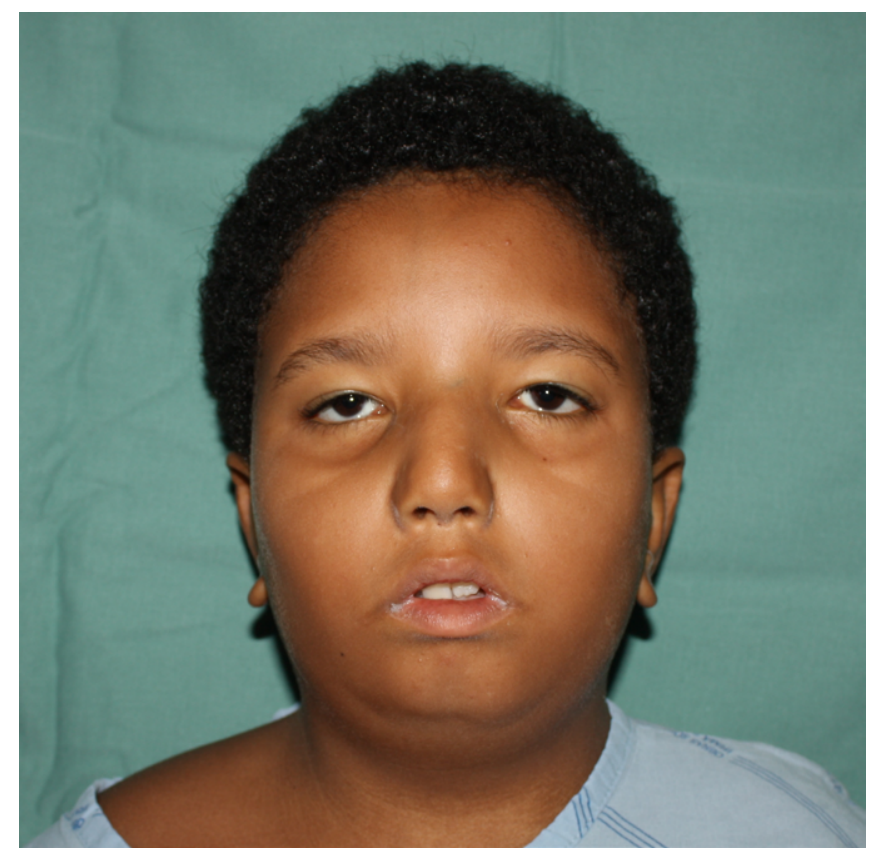

Figura 1: Vista frontal. Nota-se ausência de expressão facial. 


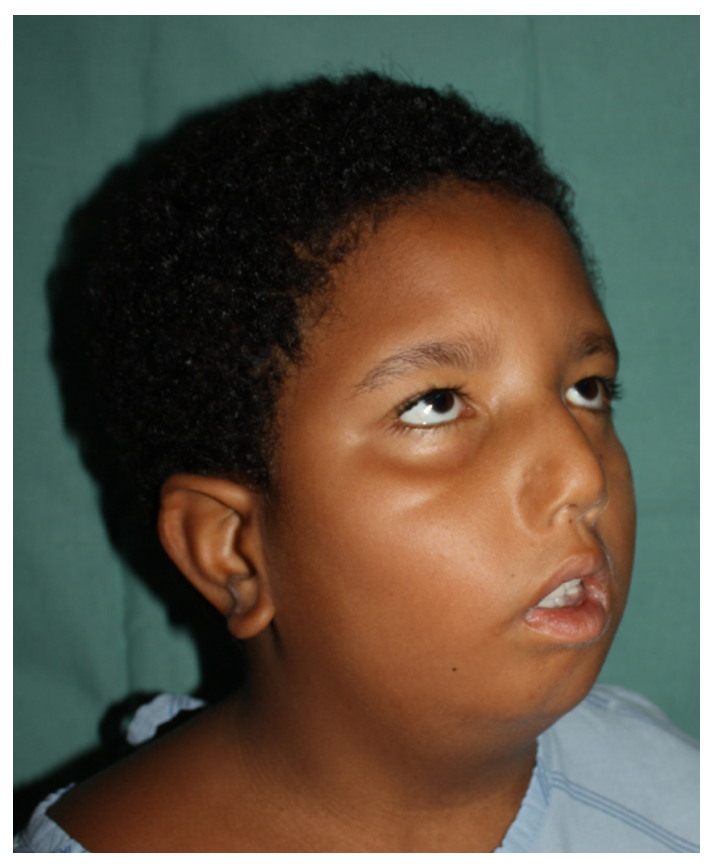

Figura 2: Ausência de selamento labial e microstomia.

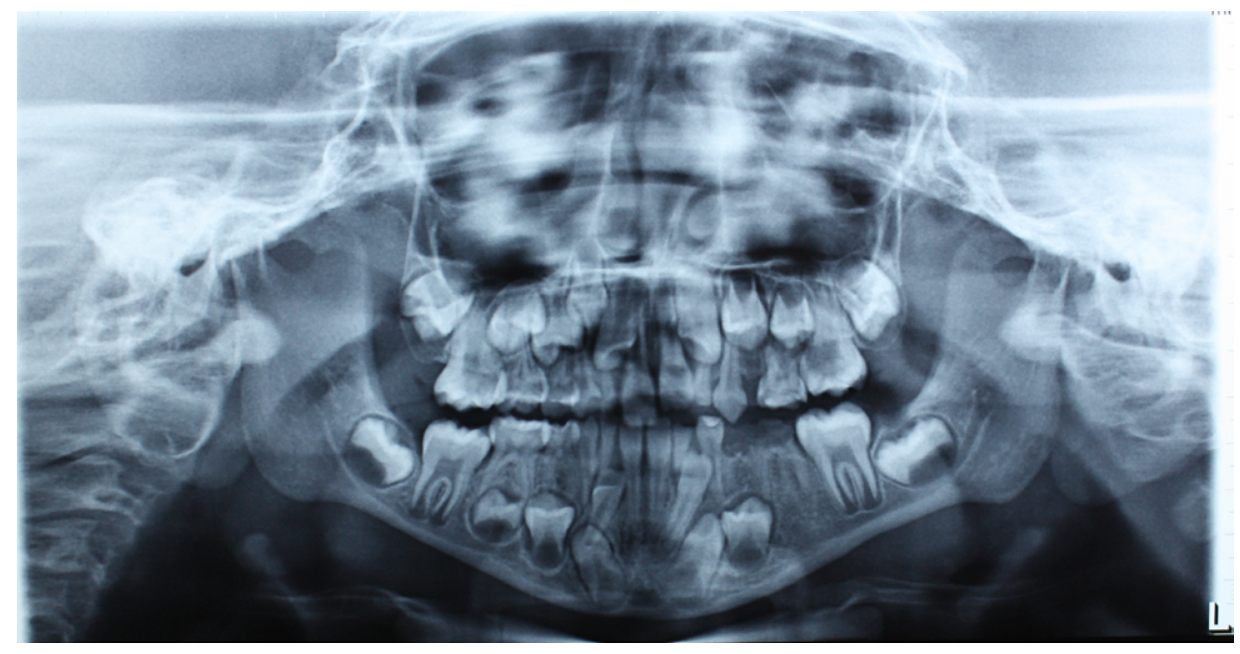

Figura 3: Radiografia panorâmica evidenciando dentição mista e presença de unidades decíduas cariadas.

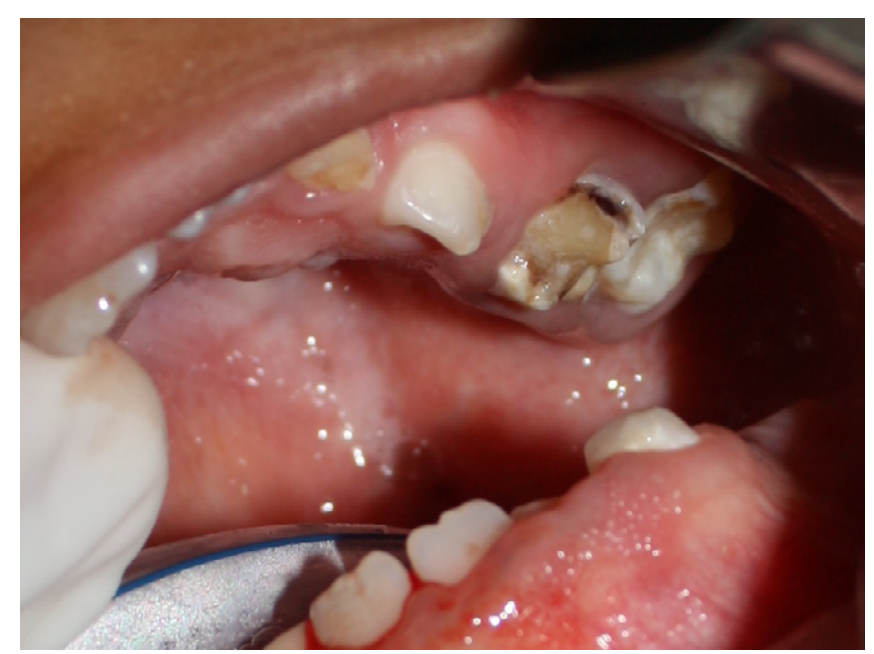

Figura 4: Paciente sob anestesia geral em transcirúrgico, observa-se múltiplas unidades cariadas. 


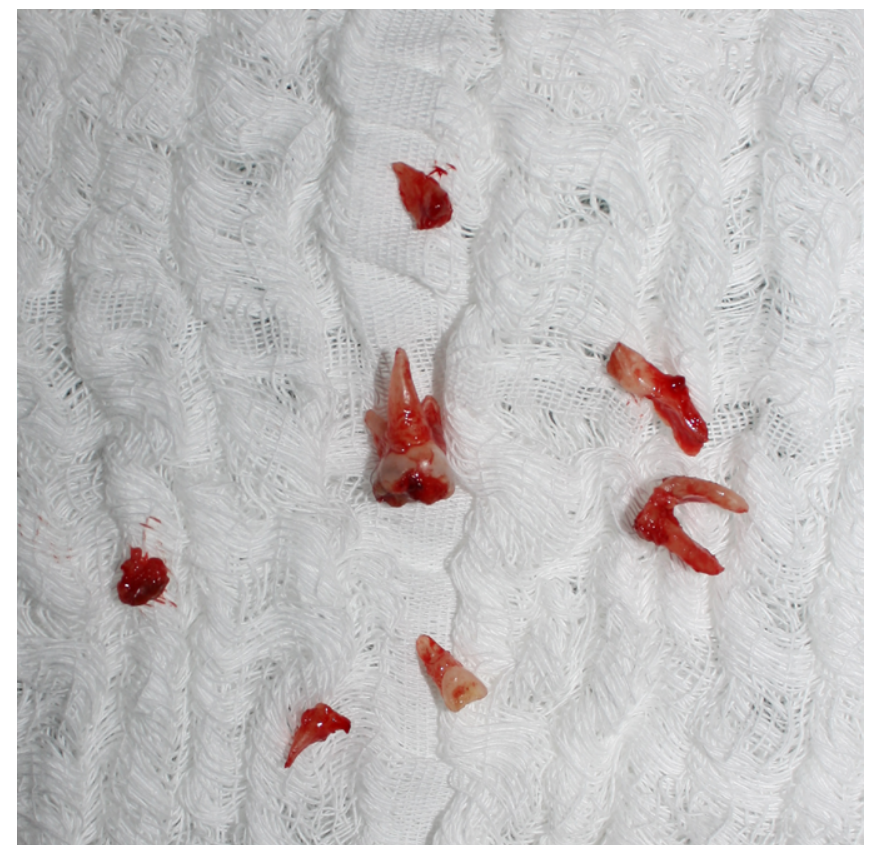

Figura 5: Unidades dentárias removidas.

\section{DISCUSSÃO}

A síndrome de Moebius ou sequência de Moebius (SM), como preferem alguns autores ${ }^{4}$, uma vez que se trata de uma entidade com múltiplas etiologias possíveis, tem como principal manifestação a paralisia uni ou bilateral do nervo facial, que confere inatividade dos músculos da expressão facial por ele inervados. ${ }^{13} \mathrm{O}$ paciente descrito nesse caso apresenta sinais característicos da entidade em discussão: ausência de mímica facial, déficit motor ocular, hipertelorismo, hipoacusia bilateral, pé direito torto, ausência de selamento labial, sialorreia, micrognatia, retrognatia, fissura palatina e miscrostomia, que corroboram com a literatura

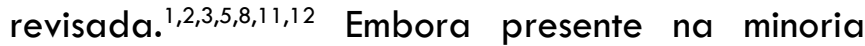
dos casos, o paciente descrito apresenta retardo no desenvolvimento cognitivo e psicomotor. ${ }^{5,11}$ Para Gondipalli e Tobias (2006) a micrognatia pode estar relacionada à deficiência neuromuscular que limita os movimentos da mandíbula e reduz 0 estímulo para o seu crescimento.

Sem causa química, física ou traumática relatada pela genitora, o sangramento ocorrido durante a gestação reforça a hipótese de que a SM pode ser causada por interrupção do aporte sanguíneo para as células fetais. ${ }^{1,2,3,5,7,8,9,12}$ Autores sugerem que a isquemia consequente pode causar agenesia, atrofia, hipoplasia ou displasia dos núcleos de nervos cranianos envolvidos na SM..$^{5,12}$
No exame intraoral foram constatadas hiperplasia gengival generalizada e lesões de cárie em dentes decíduos, assim como Scarpelli e colaboradores (2008) encontraram um quadro clínico de lesões cariosas em molares e caninos decíduos, propiciadas por peculiaridades da SM (atrofia da língua, deficiência dos músculos mastigatórios, higiene oral inadequada) e dificuldades no tratamento devido a limitação na abertura bucal e a pouca cooperação do paciente pediátrico. $\mathrm{O}$ ambiente bucal torna-se favorável ao desenvolvimento da cárie e da doença periodontal graças à dificuldade no autocuidado por falta de coordenação motora e/ou por retardo cognitivo, e à precária higienização pelo responsável devido a microstomia, aliadas ao maior tempo de permanência do alimento em contato com os dentes por causa da disfagia. ${ }^{13}$

No presente caso, por se tratar de paciente pediátrico não colaborativo e com abertura bucal limitada, foi instituída a abordagem terapêutica em ambiente hospitalar sob anestesia geral, assim como alguns autores também procederam para o tratamento da cárie e da doença periodontal em paciente adulto não cooperativo. ${ }^{13}$ Eles ainda destacaram a importância dos responsáveis pelo paciente estarem esclarecidos quanto aos riscos do procedimento e quanto à necessidade de traqueostomia para manutenção da via aérea, principalmente se houver histórico de doença respiratória. 
As malformações craniofaciais do portador de SM podem tornar a intubação endotraqueal um desafio durante atendimento sob anestesia geral. ${ }^{13,15} \mathrm{Na}$ ausência de uma traqueostomia, a abordagem usual para a criança com uma via aérea potencialmente difícil deve ser seguida, incluindo a manutenção da ventilação espontânea e a abstenção do uso de bloqueadores neuromusculares, pelo menos até ventilação com máscara ser estabelecida de forma adequada. Meios alternativos de gestão das vias aéreas (por exemplo, máscara laríngea e broncoscópios com fibra óptica) devem estar prontamente disponíveis para facilitar a intubação endotraqueal. ${ }^{13,15}$ Pacientes com SM tem mais propensão à aspiração de conteúdos para o pulmão devido à disfagia e ao fechamento incorreto da glote em casos de paralisia laríngea, devendo, portanto, ocorrer monitoramento pós-operatório da permeabilidade das vias aéreas. ${ }^{15}$

As complicações decorrentes do procedimento de anestesia geral e sedação podem estar relacionadas à hipoventilação alveolar congênita, secundária a hipoplasia ou necrose dos centros respiratórios na ponte encefálica e na medula, e a hipotonia que afeta as vias aéreas superiores, diafragma e músculos torácicos. Quando fármacos depressores do sistema nervoso central são administrados nesses pacientes, um quadro de insuficiência respiratória pode se desenvolver. ${ }^{15} \mathrm{Em}$ relato de caso, Ha e colaboradores (2003) descrevem perda de via aérea, com falha na ventilação, durante procedimento odontológico realizado sob anestesia geral em paciente com SM pouco colaborativo aos 13 anos, com posterior parada cardíaca e necessidade de traqueostomia de emergência. Aos 18 anos, apresentando novamente necessidade de tratamento odontológico, após diagnóstico de traqueomalácia, o atendimento foi realizado sob anestesia geral com traqueostomia eletiva. No presente relato, o procedimento odontológico foi realizado com o paciente intubado por via orotraqueal, sem intercorrências.

Quanto à paralisia facial, o tratamento cirúrgico ideal ainda está em discussão, sendo que a transferência microneurovascular de enxerto autógeno de músculo e tecido nervoso é atualmente o procedimento de escolha. Neste caso, o músculo risório é preferido pela facilidade de acesso e vascularização adequada. ${ }^{1,4}$ Para tratamento das maloclusões, existem poucos estudos que evidenciem um protocolo terapêutico. Foram achados na literatura desde mentoplastia, rotação anti-horária mandibular e reposicionamento do músculo mentoniano para correção ortognática e para amenizar a deficiência no selamento labial em paciente portador de SM do tipo Classe II de Angle, até a realização de distração osteogênica mandibular em paciente com retrognatismo mandibular acentuado com o intuito de dispensar meio invasivo de ventilação (traqueostomia), evitar a obstrução das vias áreas, melhorar o padrão respiratório e conferir autonomia nutricional. ${ }^{4,6}$ Nenhum tratamento para amenizar a retrognatia foi realizado neste caso, em virtude da ausência de episódios de apneia, aguardando o final do crescimento para possível cirurgia ortognática.

Apesar de ser referido por vários autores como uma manifestação bucal presente na $S M$, o tratamento da fissura labiopalatina não foi descrito na literatura revisada. ${ }^{1,2,3,8}$ No presente caso foi realizado fechamento da fissura pós-forame.

O cirurgião-dentista deve direcionar esforços na orientação em saúde bucal e prevenção de riscos, com o intuito de que estes pacientes sejam tratados em centro cirúrgico somente quando estritamente necessário. É essencial expor ao portador de SM e aos seus responsáveis a importância da escovação e do uso de dentifrício fluoretado, e quando não for possível, o uso de meios adjuvantes da higiene oral, como bochechos com solução de clorexidina. ${ }^{14}$

\section{CONSIDERAÇÕES FINAIS}

A paralisia facial e as malformações orofaciais e musculoesqueléticas apresentadas por portadores da síndrome de Moebius têm uma série de implicações sobre a saúde geral e bucal. A abordagem precoce do cirurgião-dentista é fundamental para - estabelecimento de uma condição bucal que garanta melhor qualidade de vida ao paciente.

\section{REFERÊNCIAS}

1. Bianchi B, Copelli C, Ferrari S, Ferri A, Sesenna E. Facial animation in patients with Moebius and Moebius-like 
syndromes. Int J Oral Maxillofac Surg. 2010;39(1 1):10661073. doi: $10.1016 /$ i.ijom.2010.06.020

2. Escoda-Francolí J, Sánchez-Garcés MA, Gay-Escoda C. Oral implant rehabilitation in a patient with Moebius syndrome. Med Oral Patol Oral Cir Bucal. 2009;14(6):E295-8

3. Fontenelle L, Araújo APQC, Fontan RS. Síndrome de Moebius: relato de caso. Arq Neuropsiquiatr. 2001 ;59/3-

B):81 2-814. doi: 10.1590/S0004-282X2001000500031

4. Guijarro-Martínez R, Hernández-Alfaro F. Management of maxillofacial hard and soft tissue discrepancy in Möbius sequence: clinical report and review of the literature.

J Craniomaxill Surg. 2012;40(1):11-16. doi: 10.1016/i. jcms.2010.12.006

5. Kumar D. Moebius syndrome. J Med Genet. 1990;27:122126

6. Oliveira RH, Marques M, Amarante JM, Silva A. External mandibular distraction in unusual pediatric syndromes with micrognathia and airway obstruction. J Maxillofac Oral Surg. 2013;1 2(4):456-460. doi: 10.1007/s1 2663-011-0203-3

7. Picciolini O, Porro M, Cattaneo E, Castelletti S, Masera G, Mosca $\mathrm{F}$ et al. Moebius syndrome: clinical features, diagnosis, management and early intervention. Ital J Pediatr. 2016; 42(1):56. doi: $10.1186 /$ s13052-016-0256-5

8. Rizos M, Negrón RJ, Serman N. Möbius syndrome with dental involvement: a case report and literature review. Cleft Palate Craniofacial J. 1998;35(3):262-268. doi: 10.1597/1545-1569(1998)035<0262:MBSWDI>2.3.CO;2

9. Scarpelli AC, Vertchenko TB, Resende VLS, Castilho LS, Paiva SM, Pordeus IA. Möbius syndrome: a case with oral involvement. Cleft Palate Craniofacial J. 2008;45(3):319324. doi: $10.1597 / 07-084$

10. Siögreen L, Andersson-Norinder J, Jacobsson C. Development of speech, feeding, eating, and facial expression in Möbius sequence. Int J Pediatr Otorhinolaryngol. $2001 ; 60(3): 197-204$.

11. Mattana MC, Lucena LF, Ribeiro FE, Bortagaray S, Teles AR. Síndrome de Moebius-Poland: relato de caso e revisão bibliográfica. Rev AMRIGS. 2010;54(2):197-201

12. Morales-Chávez M, Ortiz-Rincones MA, Suárez-Gorrin F. Surgical techniques for smile restoration in patients with Moebius syndrome. J Clin Exp Dent. 2013;5(4):203-207. doi: $10.4317 /$ iced.51116

13. Ha CY, Messieha ZS. Management of a patient with Mobius syndrome: a case report. Space Care Dentist. 2003;23(3):111-116

14. Nussbaum BL. Dental care for patients who are unable to open their mouths. Dent Clin C Am. 2009;53(2):323-328. doi: 10.1016/i.cden.2008.12.006
15. Gondipalli P, Tobias JD. Anesthesic implication of Möbius syndrome. J Clin Anesth. 2006;18(1):55-59. doi: 10.1016/i. ¡clinane.2005.05.005 